Sādhanā Vol. 40, Part 7, October 2015, pp. 2095-2109. (C) Indian Academy of Sciences

\title{
Numerical solution of uncertain neutron diffusion equation for imprecisely defined homogeneous triangular bare reactor
}

\author{
S NAYAK and S CHAKRAVERTY* \\ Department of Mathematics, National Institute of Technology, \\ Rourkela, Odisha 769008, India \\ e-mail: sne_chak@yahoo.com; sukantgacr@gmail.com
}

MS received 18 December 2014; revised 22 May 2015; accepted 3 August 2015

\begin{abstract}
In this paper, neutron diffusion equation of a triangular homogeneous bare reactor with uncertain parameters has been investigated. Here the involved parameters viz. geometry of the reactor, diffusion coefficient and absorption coefficient, etc. are uncertain and these are considered as fuzzy. Fuzzy values are handled through limit method which was defined for interval computations. The concept of fuzziness is hybridised with traditional finite element method to propose fuzzy finite element method. The proposed fuzzy finite element method has been used to obtain the uncertain eigenvalues of the said problem. Further these uncertain eigenvalues are compared with the traditional finite element method in special cases.
\end{abstract}

Keywords. Uncertainty; fuzzy; triangular fuzzy number (TFN); finite element method; fuzzy finite element method.

\section{Introduction}

In a nuclear reactor neutrons suffer scattering collisions with the nuclei assumed to be initially stationary and as a result a typical neutron trajectory consists of a number of short straight path elements. These are the scattering free paths. The average of these is the mean free paths. It may be noted that the path of neutron after a scattering collision is not known exactly, so there may involve some uncertainty. These uncertainties are caused due to incomplete information, impreciseness, vagueness, experimental error and different operating conditions affected by the system. In this context, many researchers have proposed various techniques to investigate these uncertainties. They have used probabilistic or statistical method as a tool to handle the uncertain parameters. As such, Monte Carlo is a well-known method for random variables. Basically, Monte Carlo method is based on the statistical simulation of the random numbers generated on the basis of a specific sampling distribution and widely used by various authors to solve neutron diffusion equation with variable parameters. In this context, Nagaya et al (2010) applied

*For correspondence 
Monte Carlo method to estimate the effective delayed neutron fraction $\beta_{\text {eff }}$ and investigated neutron diffusion problem. Further, Nagaya \& Mori (2011) proposed a new method to estimate the effective delayed neutron fraction $\beta_{\text {eff }}$ in Monte Carlo calculations. In the above paper, the eigenvalue method is jointly used with the differential operator and correlated sampling techniques. Shi \& Petrovic (2011) used Monte Carlo method to solve one-dimensional twogroup problems. Sjenitzer \& Hoogenboom (2011) gave an analytical procedure to compute the variance of the neutron flux in a simple model of a fixed-source. Recently, Yamamoto (2012) investigated the neutron leakage effect specified by buckling to generate group constants for use in reactor core designs using Monte Carlo method.

In the above investigations we have observed that a good number of observed data or experimental results are needed. However, it may not be possible always to get a large number of data. As such; a great philosopher Zadeh (1965) has proposed an alternating idea viz. the fuzzy theory, which may be used to handle uncertain and imprecise variables. Accordingly, we have used interval or fuzzy parameters to take care of the uncertainty. In general traditional interval/fuzzy arithmetic are complicated to investigate the problem. The complications are occurred due to the complexity involved in multiplication and division operations. In general, it has been seen that the associativity and commutativity property are also not well-defined. As such, we have proposed an idea of interval/fuzzy arithmetic to overcome such difficulty. The concept as proposed by Chakraverty \& Nayak (2012) and Nayak \& Chakraverty (2013) for interval arithmetic and fuzzy numbers has been used here.

The main aim of our investigation is to study the neutron diffusion equation for triangular bare reactor with imprecisely defined parameters. Initially we have considered the neutron diffusion equation for crisp parameters only. In this respect, Biswas et al (1976) have discussed a method to generate stiffness matrices which is used to find the solution of multi-group diffusion equation by natural coordinate system. Azekura (1980) has also proposed a new representation of finite element solution technique for neutron diffusion equations. The author has applied the technique to two types of one-group neutron diffusion equations to test its accuracy. It reveals from the above literature that the neutron diffusion equations are solved by using finite element method in the presence of crisp parameters only.

But the presence of uncertain parameters makes the system uncertain and we get uncertain governing differential equations. As such, few works have been done to investigate heat transfer problems using interval/fuzzy numbers. Fuzzy parameters are considered to solve heat conduction problems using finite element method and we call it as Fuzzy Finite Element Method (FFEM). Bart et al (2011) solved uncertain solution of heat conduction problem. In this paper authors gave a comparison between response surface method and other methods. Recently, Chakraverty \& Nayak (2013a) also solved the interval/fuzzy distribution of effective multiplication factors and eigenvalues for a bare square homogeneous reactor.

In this paper fuzzy finite element method has been presented. In proposed fuzzy finite element method, involved fuzzy numbers are changed into intervals through $\alpha$-cut. Then these intervals are transformed into crisp form by using some transformations. Crisp representations of intervals are defined by symbolic parameterization. With the help of crisp representation for intervals, traditional interval arithmetic has modified. Then the proposed interval arithmetic is extended for fuzzy numbers and the developed fuzzy arithmetic is used as a tool for uncertain fuzzy finite element method. Consequently the above method is used to solve one group neutron diffusion equation for triangular bare reactor and the critical eigenvalues are studied in detail. It is worth mentioning that in this paper the discretization pattern of fuzzy elements is different than Chakraverty \& Nayak (2013a). And we have also studied the convergence of the uncertain eigenvalues. Finally some important conclusions of the proposed methods are encrypted and it 
is found that this method is simpler and efficient to handle. Hence it may be used as a tool to solve different types of neutron diffusion problems for various types of nuclear reactors.

This paper has been organized in the following way. In section 2, interval and fuzzy arithmetic has been discussed to handle uncertainties. In section 3, formulation of the above said problem has been presented. In view of the formulation of the problem, a case study has been provided and the proposed method is demonstrated in section 4. Further the obtained results are depicted in section 5 .

\section{Interval and fuzzy arithmetic}

Let us consider the uncertain values in interval form and the same may be written in the following way.

$$
[\underline{x}, \bar{x}]=\{x \mid x \in R, \underline{x} \leq x \leq \bar{x}\},
$$

where $\underline{x}$ and $\bar{x}$ are lower and upper values of the interval, $m=\frac{x+\bar{x}}{2}$ and $w=\bar{x}-\underline{x}$ are centre and width of the interval $[\underline{x}, \bar{x}]$ respectively.

Let us assume $[\underline{x}, \bar{x}]$ and $[\underline{y}, \bar{y}]$ be two intervals then we have usual interval arithmetic as,

1. $[\underline{x}, \bar{x}]+[\underline{y}, \bar{y}]=[\underline{x}+\underline{y}, \bar{x}+\bar{y}]$

2. $[\underline{x}, \bar{x}]-[\underline{y}, \bar{y}]=[\underline{x}-\bar{y}, \bar{x}-\underline{y}]$

3. $[\underline{x}, \bar{x}] \times[\underline{y}, \bar{y}]=[\min \{\underline{x} \underline{y}, \underline{x} \bar{y}, \bar{x} \underline{y}, \bar{x} \bar{y}\}, \max \{\underline{x} \underline{y}, \underline{x} \bar{y}, \bar{x} \underline{y}, \bar{x} \bar{y}\}]$

4. $[\underline{x}, \bar{x}] \div[\underline{y}, \bar{y}]=[\min \{\underline{x} \div \underline{y}, \underline{x} \div \bar{y}, \bar{x} \div \underline{y}, \bar{x} \div \bar{y}\}, \max \{\underline{x} \div \underline{y}, \underline{x} \div \bar{y}, \bar{x} \div \underline{y}, \bar{x} \div \bar{y}\}]$.

The above interval arithmetic has now been extended into various fuzzy numbers viz. triangular and trapezoidal fuzzy numbers, etc. These fuzzy numbers are transformed into interval numbers by using $\alpha$-cut technique. Now the transformed intervals involve left and right continuous linear functions. Finally a fuzzy number may be represented as an ordered pair form $[f(\alpha), \bar{f}(\alpha)], 0 \leq \alpha \leq 1$ where $f(\alpha)$ and $\bar{f}(\alpha)$ are left and right monotonic increasing and decreasing functions over $[0,1]$ respectively.

Let us consider two fuzzy numbers $x=[\underline{x}(\alpha), \bar{x}(\alpha)]$ and $y=[y(\alpha), \bar{y}(\alpha)]$, and a scalar $k$ then

(i) $x=y$ if and only if $\underline{x}(\alpha)=y(\alpha)$ and $\bar{x}(\alpha)=\bar{y}(\alpha)$.

(ii) $x+y=[\underline{x}(\alpha)+\underline{y}(\bar{\alpha}), \bar{x}(\alpha) \bar{y}+\bar{y}(\alpha)]$.

(iii) $k x= \begin{cases}{[k \underline{x}(\alpha), k \overline{\bar{x}}(\alpha)],} & k \geq 0 \\ {[k \bar{x}(\alpha), k \underline{x}(\alpha)],} & k<0\end{cases}$

Definition 2.1

Above interval arithmetic for real interval are defined here as follows (Chakraverty \& Nayak 2012)

1. $[\underline{x}, \bar{x}]+[\underline{y}, \bar{y}]=\left[\min \left\{\lim _{n \rightarrow \infty} l_{1}+\lim _{n \rightarrow \infty} l_{2}, \lim _{n \rightarrow 1} l_{1}+\lim _{n \rightarrow 1} l_{2}\right\}, \max \left\{\lim _{n \rightarrow \infty} l_{1}+\lim _{n \rightarrow \infty} l_{2}, \lim _{n \rightarrow 1} l_{1}+\lim _{n \rightarrow 1} l_{2}\right\}\right]$ 
2. $[\underline{x}, \bar{x}]-[\underline{y}, \bar{y}]=\left[\min \left\{\lim _{n \rightarrow \infty} l_{1}-\lim _{n \rightarrow 1} l_{2}, \lim _{n \rightarrow 1} l_{1}-\lim _{n \rightarrow \infty} l_{2}\right\}, \max \left\{\lim _{n \rightarrow \infty} l_{1}-\lim _{n \rightarrow 1} l_{2}, \lim _{n \rightarrow 1} l_{1}-\lim _{n \rightarrow \infty} l_{2}\right\}\right]$

3. $[\underline{x}, \bar{x}] \times[\underline{y}, \bar{y}]=\left[\min \left\{\lim _{n \rightarrow \infty} l_{1} \times \lim _{n \rightarrow \infty} l_{2}, \lim _{n \rightarrow 1} l_{1} \times \lim _{n \rightarrow 1} l_{2}\right\}, \max \left\{\lim _{n \rightarrow \infty} l_{1} \times \lim _{n \rightarrow \infty} l_{2}, \lim _{n \rightarrow 1} l_{1} \times \lim _{n \rightarrow 1} l_{2}\right\}\right]$

4. $[\underline{x}, \bar{x}] \div[\underline{y}, \bar{y}]=\left[\min \left\{\lim _{n \rightarrow \infty} l_{1} \div \lim _{n \rightarrow 1} l_{2}, \lim _{n \rightarrow 1} l_{1} \div \lim _{n \rightarrow \infty} l_{2}\right\}, \max \left\{\lim _{n \rightarrow \infty} l_{1} \div \lim _{n \rightarrow 1} l_{2}, \lim _{n \rightarrow 1} l_{1} \div \lim _{n \rightarrow \infty} l_{2}\right\}\right]$,

where for an arbitrary interval $[\underline{a}, \bar{a}]=\left\{\underline{a}+\frac{w}{n}=l \mid \underline{a} \leq l \leq \bar{a}, n \in[1, \infty)\right\}$ and $w=\bar{a}-\underline{a}$ is the width of the interval.

Definition 2.2

A fuzzy number $\tilde{A}=\left[a^{L}, a^{N}, a^{R}\right]$ is said to be triangular fuzzy number (figure 1) when the membership function is given by

$$
\mu_{\tilde{A}}(x)= \begin{cases}0, & x \leq a^{L} ; \\ \frac{x-a^{L}}{a^{N}-a^{L}}, & a^{L} \leq x \leq a^{N} ; \\ \frac{a^{R}-x}{a^{R}-a^{N}}, & a^{N} \leq x \leq a^{R} ; \\ 0, & x \geq a^{R} .\end{cases}
$$

\section{Definition 2.3}

The triangular fuzzy number $\tilde{A}=\left[a^{L}, a^{N}, a^{R}\right]$ may be transformed into interval form by using $\alpha$-cut in the following form

$$
\tilde{A}=\left[a^{L}, a^{N}, a^{R}\right]=\left[a^{L}+\left(a^{N}-a^{L}\right) \alpha, a^{R}-\left(a^{R}-a^{N}\right) \alpha\right],
$$

\section{Definition 2.4}

If the fuzzy numbers are taken in interval form then using Definition 2.1, the arithmetic rules may be defined as

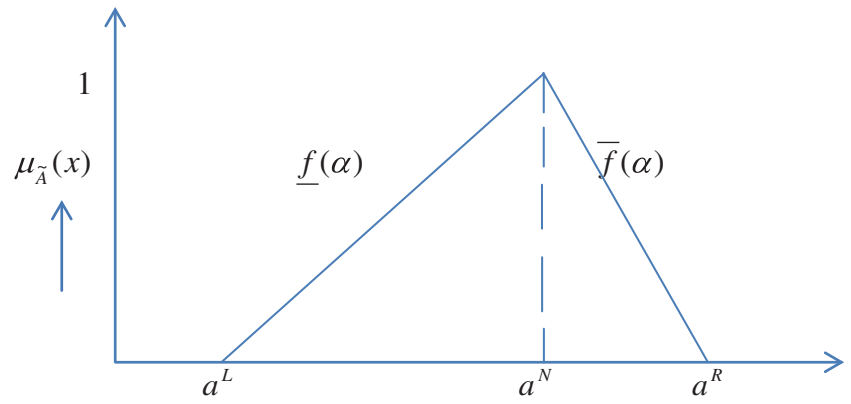

Figure 1. Triangular fuzzy number (TFN). 
1. $[\underline{x}(\alpha), \bar{x}(\alpha)]+[\underline{y}(\alpha), \bar{y}(\alpha)]$

$=\left[\min \left\{\lim _{n \rightarrow \infty} m_{1}+\lim _{n \rightarrow \infty} m_{2}, \lim _{n \rightarrow 1} m_{1}+\lim _{n \rightarrow 1} m_{2}\right\}, \max \left\{\lim _{n \rightarrow \infty} m_{1}+\lim _{n \rightarrow \infty} m_{2}, \lim _{n \rightarrow 1} m_{1}+\lim _{n \rightarrow 1} m_{2}\right\}\right]$

2. $[\underline{x}(\alpha), \bar{x}(\alpha)]-[\underline{y}(\alpha), \bar{y}(\alpha)]$

$=\left[\min \left\{\lim _{n \rightarrow \infty} m_{1}-\lim _{n \rightarrow 1} m_{2}, \lim _{n \rightarrow 1} m_{1}-\lim _{n \rightarrow \infty} m_{2}\right\}, \max \left\{\lim _{n \rightarrow \infty} m_{1}-\lim _{n \rightarrow 1} m_{2}, \lim _{n \rightarrow 1} m_{1}-\lim _{n \rightarrow \infty} m_{2}\right\}\right]$

3. $[\underline{x}(\alpha), \bar{x}(\alpha)] \times[\underline{y}(\alpha), \bar{y}(\alpha)]$

$=\left[\min \left\{\lim _{n \rightarrow \infty} m_{1} \times \lim _{n \rightarrow \infty} m_{2}, \lim _{n \rightarrow 1} m_{1} \times \lim _{n \rightarrow 1} m_{2}\right\}, \max \left\{\lim _{n \rightarrow \infty} m_{1} \times \lim _{n \rightarrow \infty} m_{2}, \lim _{n \rightarrow 1} m_{1} \times \lim _{n \rightarrow 1} m_{2}\right\}\right]$

4. $[\underline{x}(\alpha), \bar{x}(\alpha)] \div[\underline{y}(\alpha), \bar{y}(\alpha)]$

$=\left[\min \left\{\lim _{n \rightarrow \infty} m_{1} \div \lim _{n \rightarrow 1} m_{2}, \lim _{n \rightarrow 1} m_{1} \div \lim _{n \rightarrow \infty} m_{2}\right\}, \max \left\{\lim _{n \rightarrow \infty} m_{1} \div \lim _{n \rightarrow 1} m_{2}, \lim _{n \rightarrow 1} m_{1} \div \lim _{n \rightarrow \infty} m_{2}\right\}\right]$,

where for any arbitrary interval

$$
[\underline{f}(\alpha), \bar{f}(\alpha)]=\left\{\underline{f}(\alpha)+\frac{\bar{f}(\alpha)-\underline{f}(\alpha)}{n}=m \mid \underline{f}(\alpha) \leq m \leq \bar{f}(\alpha), n \in[1, \infty)\right\} .
$$

While solving above said problems by traditional FEM we usually consider the associated parameters and formulations in crisp form. But, to handle the uncertainty while using FEM, we must formulate the FEM in uncertain form (fuzzy or interval) using the fuzzy or interval computation. In this paper we formulate the problem first as traditional FEM for the sake of completeness. Then the problem has been formulated considering the uncertainty. Henceforth the main aim of this paper is to solve the uncertain one group neutron diffusion equation for triangular bare reactor by an alternate non-probabilistic method presented and we call it FFEM or IFEM.

\section{Formulation of the problem}

As it is known that the principle of neutron conservation can be expressed in a simpler form for a system of mono-energetic neutrons. Whereas, multi-group equations can be analysed by considering the series of one group equations.

The standard functional for corresponding one group diffusion equation may be written as

$$
I(\phi)=\frac{1}{2} \iint_{R}\left[D\left(\frac{\partial \phi}{\partial x}\right)^{2}+D\left(\frac{\partial \phi}{\partial y}\right)^{2}+\sigma \phi^{2}-2 S \phi\right] d x d y
$$

where $\phi$ is a constant over a partial/total portion of the periphery, $D$ is the diffusion coefficient, $\sigma$ is the absorption coefficient and $S$ is the source term.

If we apply traditional FEM to handle the problem the corresponding domain of the problem is divided into number of subdomain and each of them is called element. For each element we may find the functional and similarly for the entire domain the functional may be found out by summing each functional element wise. The above procedure may be written in the following way. 
Let us consider the domain $R$ which may be represented as

$$
R=\sum_{e=1}^{n} R^{e}
$$

and the functional $I(\phi)$ is defined as

$$
I(\phi)=\sum_{e=1}^{n} I^{e}(\phi)
$$

where $n$ is the total number of elements and $I^{e}(\phi)$ denotes the contribution of element $e$ to the functional $I(\phi)$. Now Eq. (9) for each elemental functional may be written as

$$
I^{e}(\phi)=\frac{1}{2} \iint_{R}\left[D^{e}\left(\frac{\partial \phi^{e}}{\partial x}\right)^{2}+D^{e}\left(\frac{\partial \phi^{e}}{\partial y}\right)^{2}+\sigma^{e} \phi^{2}-2 S^{e} \phi^{e}\right] d x d y .
$$

For each element $e$ the scalar flux $\phi^{e}$ is approximated by a piecewise interpolation polynomial. Depending on the interpolation polynomial, stiffness matrices are obtained by minimizing the elemental functional $I^{e}(\phi)$. The stiffness matrices are assembled and finally we get the algebraic form which is represented as

$$
[K]\{\phi\}=\{Q\},
$$

where $[K]$ is the assembled stiffness matrix corresponding to the leakage and absorption terms and $\{Q\}$ is the assembled force vector for the source term.

In general when neutrons undergo scattering, the neutron transport equation involves uncertainty. These uncertainties are occurred due to the imprecise value of operating parameters viz. geometry, diffusion and absorption coefficients, etc. Here uncertain parameters are considered as fuzzy and transformed into interval through $\alpha$-cut. To investigate the uncertain spectrum of neutron flux distribution we have formulated fuzzy finite element method with linear triangular fuzzy element discretizing the domain.

Let us consider that the coordinates of linear triangular elements are in fuzzy and hence we may write

$$
\begin{aligned}
& \tilde{x}=\tilde{L}_{1} \tilde{x}_{1}+\tilde{L}_{2} \tilde{x}_{2}+\tilde{L}_{3} \tilde{x}_{3} ; \\
& \tilde{y}=\tilde{L}_{1} \tilde{y}_{1}+\tilde{L}_{2} \tilde{y}_{2}+\tilde{L}_{3} \tilde{y}_{3} ; \\
& \tilde{L}=\tilde{L}_{1}+\tilde{L}_{2}+\tilde{L}_{3} ;
\end{aligned}
$$

where $\tilde{L}_{i}(i=1,2,3)$ are nondimensionalized fuzzy coordinates which may be changed into intervals.

The above Eq. (14) in matrix form is represented in the following way

$$
\begin{aligned}
& {\left[\begin{array}{ccc}
1 & 1 & 1 \\
\tilde{x}_{1} & \tilde{x}_{2} & \tilde{x}_{3} \\
\tilde{y}_{1} & \tilde{y}_{2} & \tilde{y}_{3}
\end{array}\right]\left\{\begin{array}{c}
\tilde{L}_{1} \\
\tilde{L}_{2} \\
\tilde{L}_{3}
\end{array}\right\}=\left\{\begin{array}{c}
1 \\
\tilde{x} \\
\tilde{y}
\end{array}\right\}} \\
& \Rightarrow\left\{\begin{array}{c}
\tilde{L}_{1} \\
\tilde{L}_{2} \\
\tilde{L}_{3}
\end{array}\right\}=\left[\begin{array}{ccc}
1 & 1 & 1 \\
\tilde{x}_{1} & \tilde{x}_{2} & \tilde{x}_{3} \\
\tilde{y}_{1} & \tilde{y}_{2} & \tilde{y}_{3}
\end{array}\right]^{-1}\left\{\begin{array}{c}
1 \\
\tilde{x} \\
\tilde{y}
\end{array}\right\}
\end{aligned}
$$




$$
\Rightarrow\left\{\begin{array}{c}
\tilde{L}_{1} \\
\tilde{L}_{2} \\
\tilde{L}_{3}
\end{array}\right\}=\left[\begin{array}{lll}
\tilde{x}_{2} \tilde{y}_{3}-\tilde{x}_{3} \tilde{y}_{2} & \tilde{y}_{2}-\tilde{y}_{3} & \tilde{x}_{3}-\tilde{x}_{2} \\
\tilde{x}_{3} \tilde{y}_{1}-\tilde{x}_{1} \tilde{y}_{3} & \tilde{y}_{3}-\tilde{y}_{1} & \tilde{x}_{1}-\tilde{x}_{3} \\
\tilde{x}_{1} \tilde{y}_{2}-\tilde{x}_{2} \tilde{y}_{1} & \tilde{y}_{1}-\tilde{y}_{2} & \tilde{x}_{2}-\tilde{x}_{1}
\end{array}\right]\left\{\begin{array}{c}
1 \\
\tilde{x} \\
\tilde{y}
\end{array}\right\}
$$

where area of the fuzzy triangle is $\tilde{\Delta}=\frac{1}{2}\left[\begin{array}{ccc}1 & 1 & 1 \\ \tilde{x}_{1} & \tilde{x}_{2} & \tilde{x}_{3} \\ \tilde{y}_{1} & \tilde{y}_{2} & \tilde{y}_{3}\end{array}\right]$.

We now denote

$$
\begin{aligned}
& \tilde{a}_{1}=\tilde{x}_{3}-\tilde{x}_{2}, \tilde{a}_{2}=\tilde{x}_{1}-\tilde{x}_{3}, \tilde{a}_{3}=\tilde{x}_{2}-\tilde{x}_{1} ; \\
& \tilde{b}_{1}=\tilde{y}_{2}-\tilde{y}_{3}, \tilde{b}_{2}=\tilde{y}_{3}-\tilde{y}_{1}, \tilde{b}_{3}=\tilde{y}_{1}-\tilde{y}_{2} ; \\
& \tilde{c}_{1}=\tilde{x}_{2} \tilde{y}_{3}-\tilde{x}_{3} \tilde{y}_{2}, \tilde{c}_{2}=\tilde{x}_{3} \tilde{y}_{1}-\tilde{x}_{1} \tilde{y}_{3}, \tilde{c}_{3}=\tilde{x}_{1} \tilde{y}_{2}-\tilde{x}_{2} \tilde{y}_{1} .
\end{aligned}
$$

If $\tilde{\phi}$ is the flux distribution then it may be written as

$$
\tilde{\phi}=\tilde{L}_{1} \tilde{\phi}_{1}+\tilde{L}_{2} \tilde{\phi}_{2}+\tilde{L}_{3} \tilde{\phi}_{3} .
$$

The differentiation and integration formulae may be seen in Chakraverty \& Nayak (2013a).

Using above formulation one may get the leakage and absorption stiffness matrices in terms of fuzzy values. With the help of proposed arithmetic these fuzzy values may be transformed into crisp numbers. Accordingly, corresponding stiffness matrices of each element for leakage and absorption term is given in Chakraverty \& Nayak (2013a).

The above discussed fuzzy arithmetic in terms of $\alpha$-cut has been used here for finite element method. Here in figure 2, a schematic diagram has presented, which gives the basic idea to encrypt the process of modified fuzzy finite element method. It involves three steps such as input, output and hidden layer. In the input step we have considered uncertain parameters and field variables. These uncertain parameters are taken as fuzzy. In hidden layer, element properties are

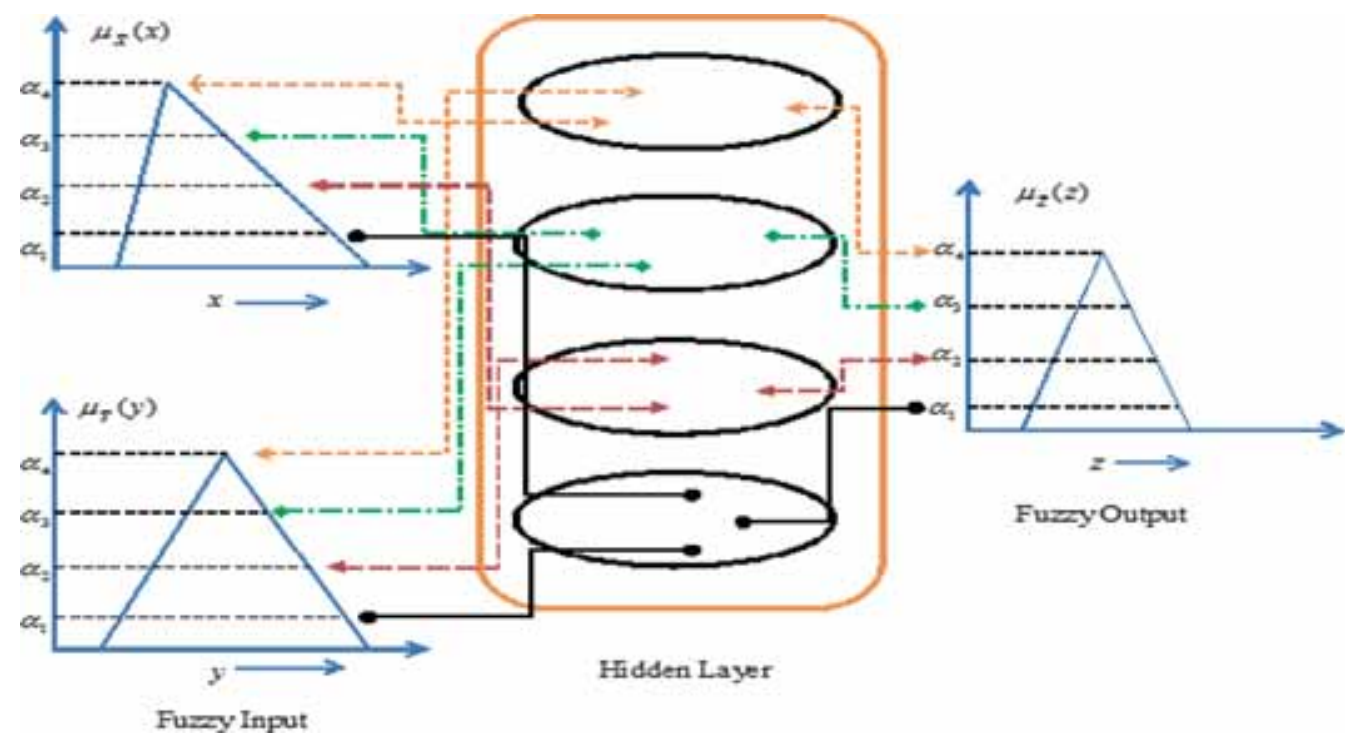

Figure 2. Model diagram of the modified fuzzy finite element procedure. 


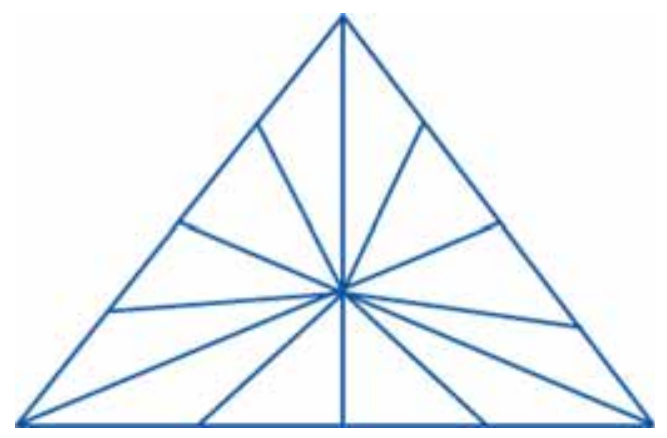

Figure 3. Triangular element discretization of triangular plate.

obtained by using various fuzzy parameters. The element properties and fuzzy stiffness matrices are assembled and finally global stiffness matrices are investigated. Further initial and boundary conditions are imposed and fuzzy system of equations are solved through the proposed technique (Chakraverty \& Nayak 2013b). Finally the executions of various sub-steps happen inside the hidden layer. Finally we get uncertain fuzzy solutions as output, which may be different in type and nature comparing with the input fuzzy parameters. Here in figure 2 we have considered triangular fuzzy numbers as input parameters. Alpha level representation of two fuzzy sets $\tilde{X}$ and $\tilde{Y}$ with their triangular membership functions for fuzzy arithmetic operation (Nagaya et al 2010 ) is shown in figure 2. Deterministic value is obtained for $\alpha_{4}$-level of fuzzy sets whereas for $\alpha_{1}, \alpha_{2}$ and $\alpha_{3}$ level we get different interval values. If we consider the alpha value as zero then deterministic interval lies on $\mathrm{X}$-axis. The output may be generated by considering all possible combinations of the alpha level.

\section{Case study}

The governing differential equation for the bare homogeneous reactor (Yamamoto 2012; Glasstone \& Sesonke 2004) is as follows

$$
D \nabla^{2} \phi+S=\Sigma_{a} \phi
$$

We have considered a triangular (equilateral) bare reactor having each side of four units and it is discretized into triangular element as given in figure 3.

Fuzzy parameters are taken for diffusion and absorption coefficients which are presented in table 1.

Initially the governing one group neutron diffusion equation is solved by considering only crisp parameters and then the proposed method for the modelled uncertain one group neutron diffusion equation is solved. Eigenvalues for both the crisp and fuzzy parameters are obtained and the values are depicted in table 2 for different number of elements in the FEM and FFEM discretization.

Table 1. Triangular fuzzy numbers for uncertain parameters.

\begin{tabular}{lcc}
\hline Parameters & Crisp value & TFN \\
\hline Diffusion coefficient & 1 & {$[0.5+0.5 \alpha, 1.5-0.5 \alpha]$} \\
Absorption coefficient & 1 & {$[0.5+0.5 \alpha, 1.5-0.5 \alpha]$} \\
\hline
\end{tabular}


Table 2. Crisp and triangular fuzzy eigenvalues for triangular plate.

\begin{tabular}{lcc}
\hline Number of elements & Crisp eigenvalues & Triangular fuzzy eigenvalues \\
\hline 6 & 0.6425 & {$[0.6377,0.6425,0.647]$} \\
12 & 0.6264 & {$[0.6236,0.6264,0.6297]$} \\
24 & 0.526 & {$[0.5251,0.526,0.527]$} \\
48 & 0.5083 & {$[0.508,0.5083,0.5087]$} \\
96 & 0.5034 & {$[0.5032,0.5034,0.5036]$} \\
192 & 0.5015 & {$[0.5015,0.5015,0.5016]$} \\
384 & 0.5007 & {$[0.5007,0.5007,0.5008]$} \\
1536 & 0.5002 & {$[0.5002,0.5002,0.5002]$} \\
\hline
\end{tabular}

For better visualization of the obtained results, eigenvalues for different number of discretizations of the domain are plotted and shown in figures 4-11.

The variation of both the crisp and fuzzy eigenvalues may be studied from figure 12 . Here a set of eigenvalues is given and the convergence is studied.

\section{Discussion}

When neutrons undergo diffusion in reactor then it suffers scattering collisions with the nuclei assumed to be initially stationary and they make different trajectory paths. Hence, to study the eigenvalue problem for corresponding one group neutron diffusion equation, we have considered triangular element discretization for one group neutron diffusion equation for a triangular bare reactor. Initially the eigenvalue problem is solved by classical finite element method for crisp parameters and then it is solved by proposed fuzzy finite element procedure.

Here triangular bare reactor is considered and neutron flux at centre of the triangular geometry is taken as zero. The geometry is discretized into number of triangular elements as given in figure 3. So the neutron flux distributions are studied for other nodal points. Solving eigenvalue

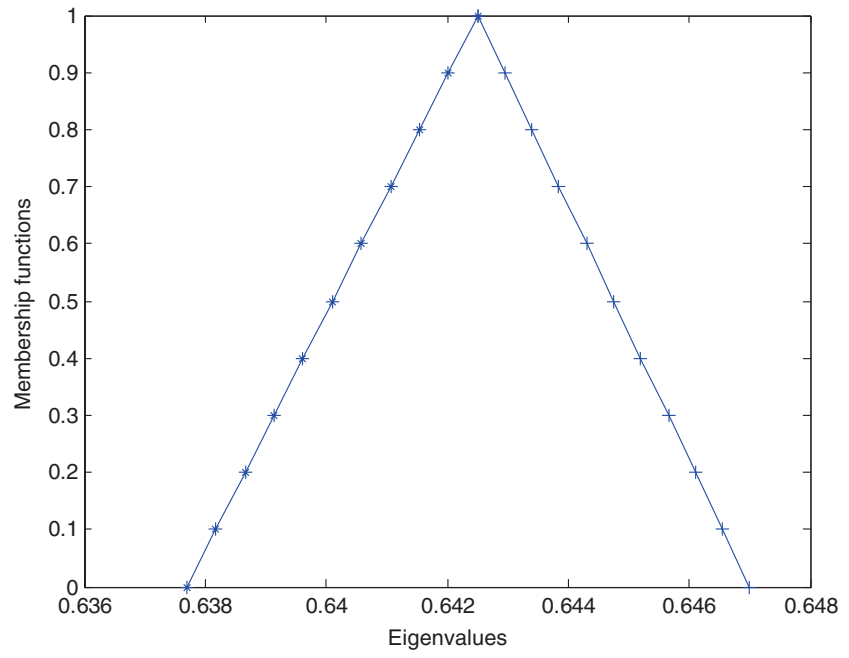

Figure 4. 6 elements discretization. 


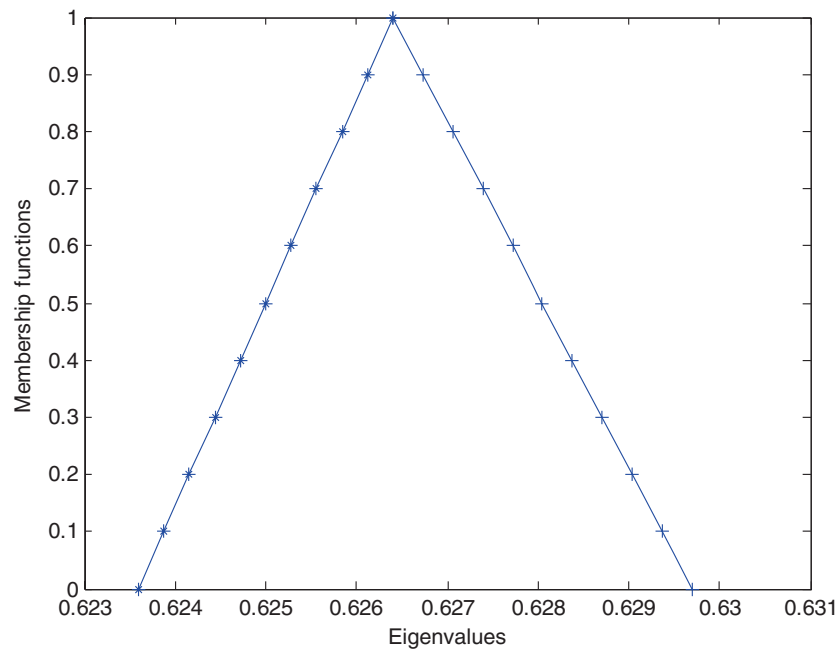

Figure 5. 12 elements discretization.

problem we get a set of eigenvalues for different number of element discretizations. It is seen that the eigenvalues are converging with respect to the increase in number of elements for discretized triangular bare reactor. The pattern of the convergence is presented in table 2 .

The diffusion and absorption coefficients are taken as the uncertain parameters. These uncertain parameters are considered as TFN to investigate the uncertain eigenvalues. Again the boundary condition for this uncertain case is taken same as that of the crisp case. As we increase the number of elements the uncertain eigenvalues gets converged. Further, uncertain width of eigenvalues decreases with increase in number of discretizations of the triangular bare homogeneous reactor.

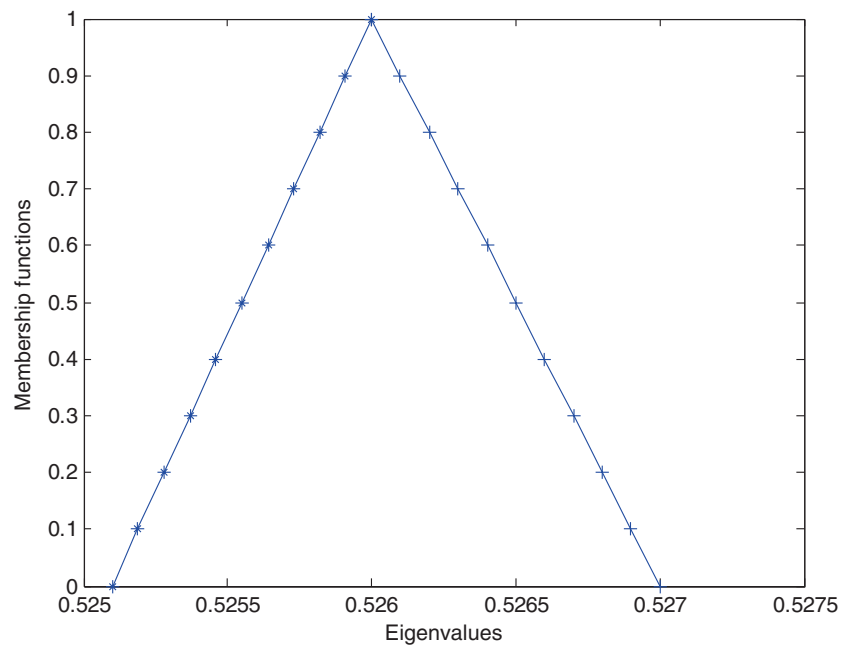

Figure 6. 24 elements discretization. 


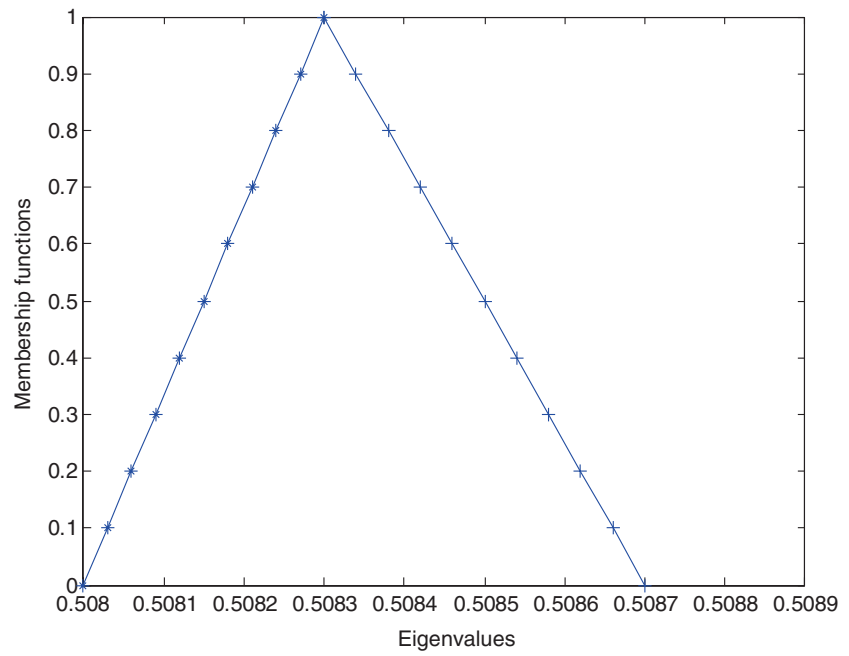

Figure 7. 48 elements discretization.

From the obtained results it is observed that the shape of the TFN changes as we discretize the domain into more number of elements and these are shown in figures 4-11. This variation of TFN occurs due to the left, right and centre values of the TFN. As we move on with the increase in number of discretization of the domain we get right angular shaped fuzzy number. The trends of the shapes are shown in figures 8-11. In figures 9 and 10 the left and centre values are obtained as same so we get left monotonically increasing function parallel to membership functions axis and width of the left bound from centre becomes zero. In figure 11, the left, right and centre values approximately coincide and hence we get a straight line parallel to membership function axis. Here the variations of eigenvalues become constant with the change of membership functions. From figure 12 it is seen that if we go on increasing the number of discretization of the said

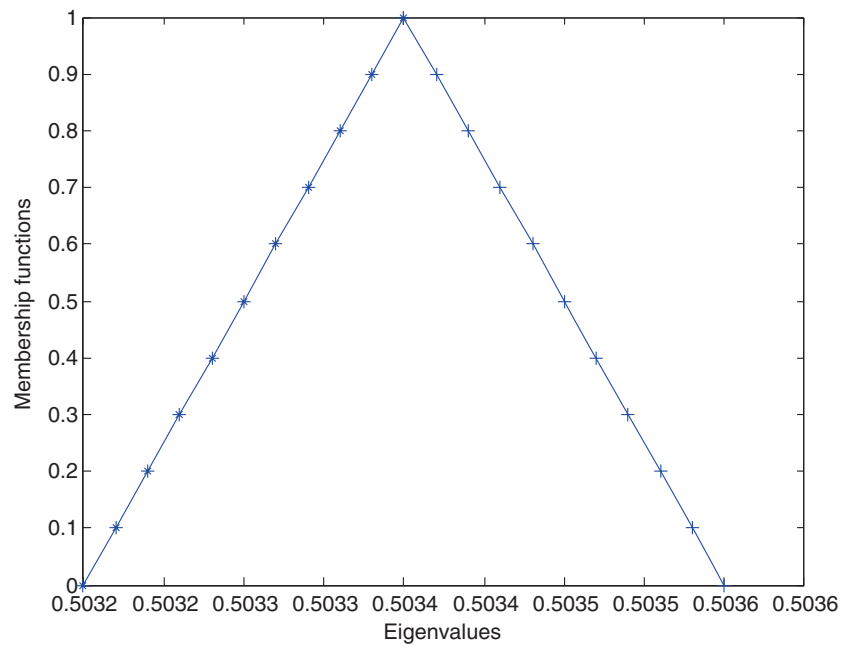

Figure 8. 96 elements discretization. 


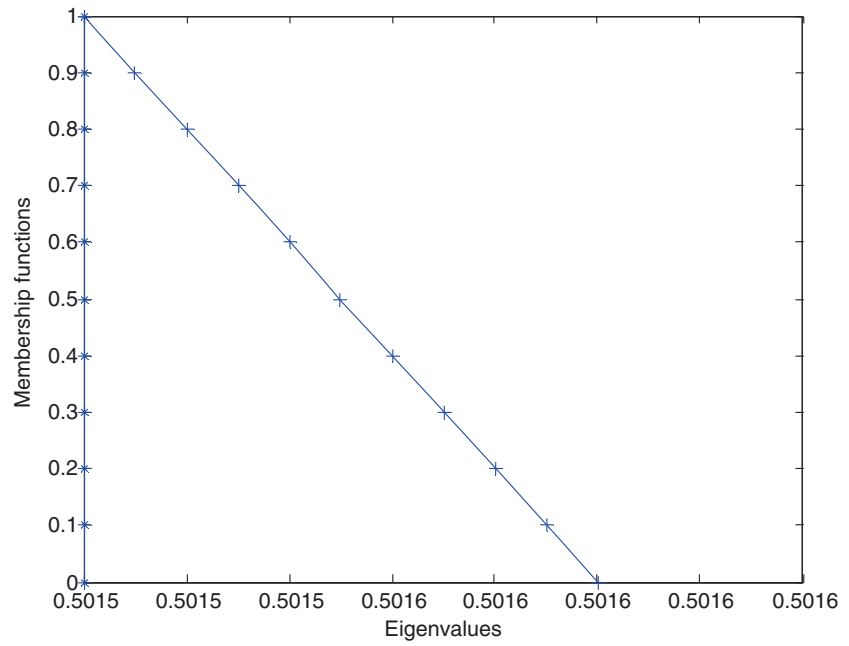

Figure 9. 192 elements discretization.

domain we get a series of uncertain eigenvalues and these triangular fuzzy eigenvalues converges to a constant value.

It may be noted that the reliability of the fuzzy results can be seen in the special cases viz. crisp and interval which are derived from the fuzzy values. As such three cases are reported with respect to the above.

Case 1. Here we have considered only left monotonic increasing functions of the resultant eigenvalues. The resulting eigenvalues vary with the value of membership functions. Assigning zero for the value of $\alpha$ we get the left bound of the uncertain fuzzy eigenvalues. Similarly if the

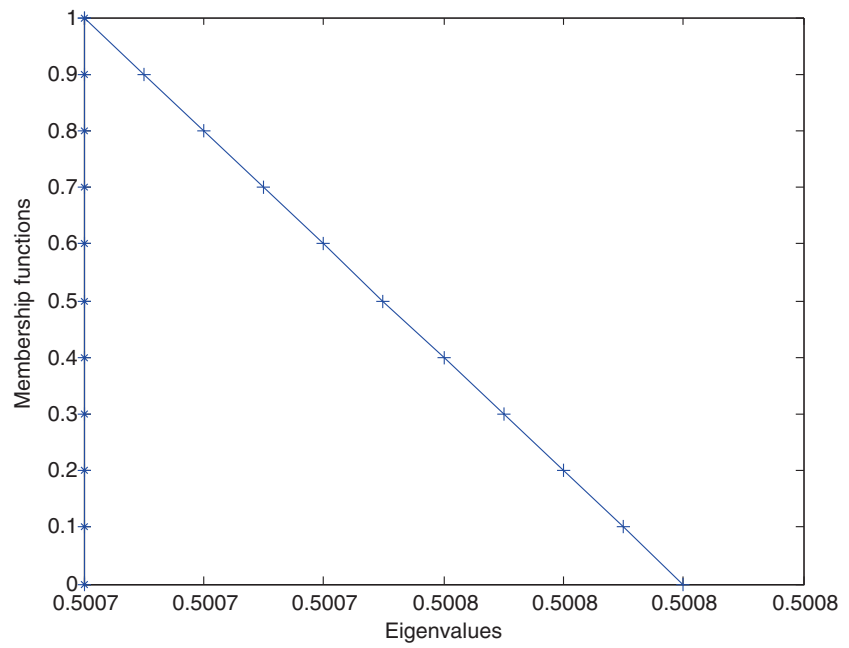

Figure 10. 384 elements discretization. 


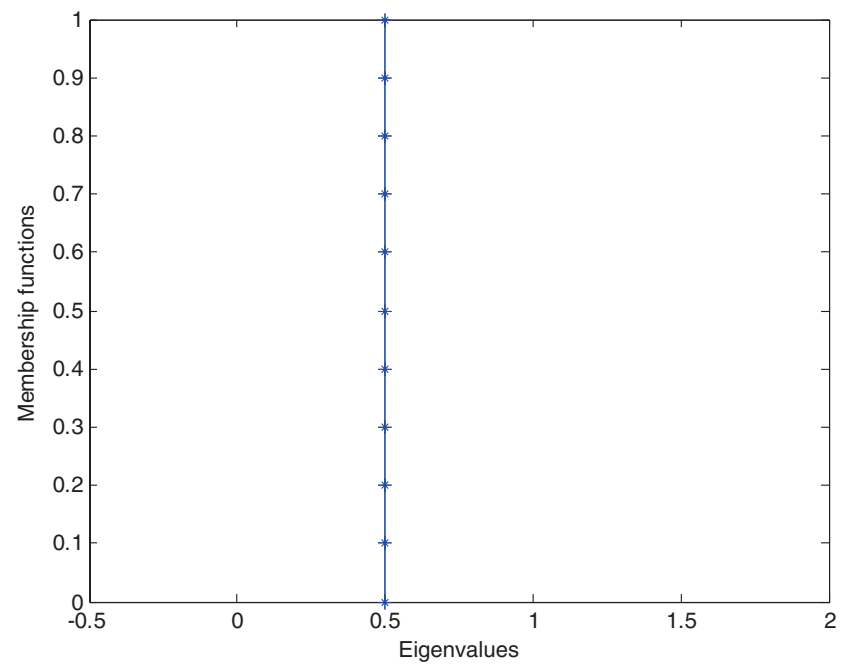

Figure 11. 1536 elements discretization.

value of $\alpha$ is taken as one then we get right bound of the left monotonic increasing functions which are the centre value of TFN.

Case 2. In this case only right monotonic decreasing functions of the resultant eigenvalues are considered. Resulting eigenvalues vary with the value of membership functions. Assigning zero for the value of $\alpha$ we get the right bound of the uncertain fuzzy eigenvalues. Similarly if the

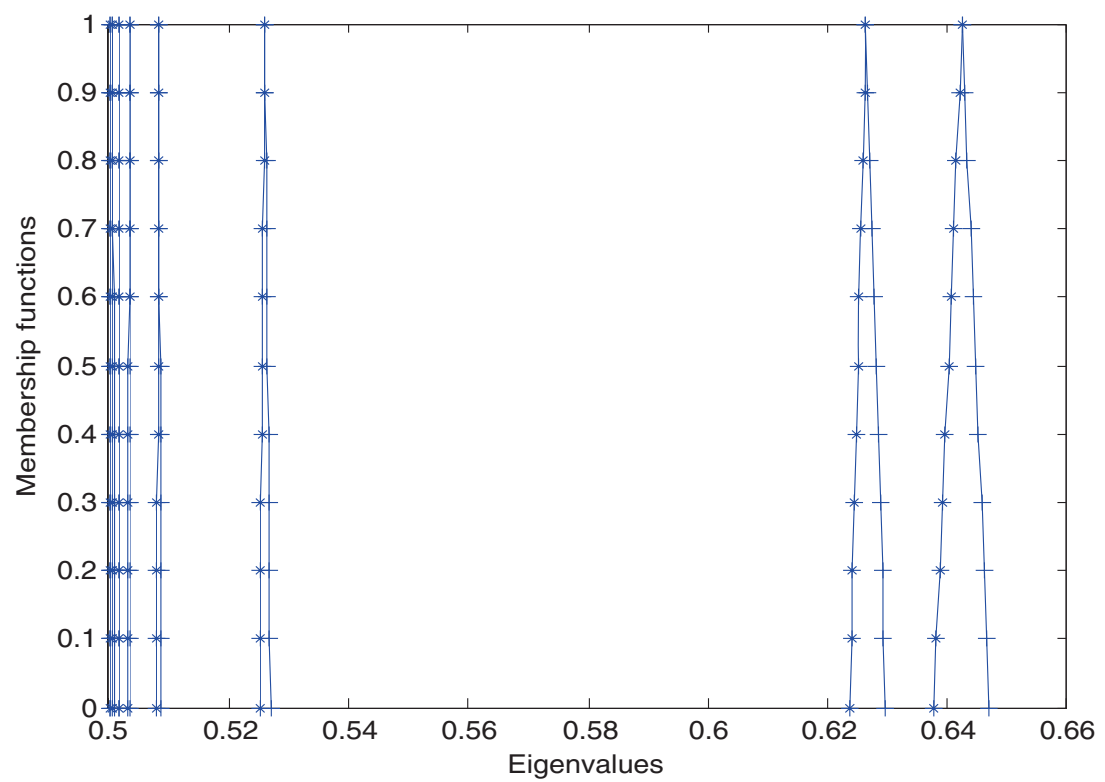

Figure 12. Triangular fuzzy membership functions for various discretizations of the domain. 
value of $\alpha$ is taken as one then we get left bound of the right monotonic decreasing functions which are the centre value of TFN.

Case 3. In this case let us consider the value of $\alpha$ is one for both the left and right monotonic functions. We observe that the resultant eigenvalues become same for both monotonic functions. If we consider TFN then we find that it is nothing but the centre value. Here we get an interval of eigenvalues where the membership functions are normalised.

\section{Conclusion}

The main purpose of this paper is to present an alternative non-probabilistic method to manage various uncertain engineering and science problems. Here the traditional interval arithmetic is modified for the said problem and a simpler method is proposed to compute interval arithmetic. Present paper gives an alternative idea about different interval arithmetic by putting the computation in a loop varying $\mathrm{n}$ and then the lower and upper values depending upon the convergence. The idea of modified interval arithmetic is then extended for uncertain fuzzy numbers. As such uncertain parameters are taken as fuzzy and the fuzzy numbers are converted into interval using $\alpha$-cut techniques. These fuzzy numbers contain left monotonically increasing and right monotonically decreasing functions respectively.

In this paper we have considered one group of neutron diffusion equation for triangular bare reactor. The corresponding eigenvalues for one group of neutron diffusion equation for bare triangular homogeneous reactor is investigated. The presence of uncertain parameters makes the system uncertain and the uncertain eigenvalues are studied in detail. To handle uncertain system, the problem is modelled and presented by a modified fuzzy finite element method. In this study we found that proposed fuzzy finite element method for triangular element discretization gives a general procedure to solve the said problem. The generalization may be extended for various types of element discretizations. This method is found to be reliable by considering the convergence of uncertain width of the obtained results. It may be concluded that the fuzzy finite element method with the proposed interval computation is simpler to handle and also efficient. Hence it may be used as a tool to solve neutron diffusion problems for various other types of nuclear reactors.

\section{Acknowledgement}

The authors would like to thank BRNS (Board of Research in Nuclear Sciences), Department of Atomic Energy, (DAE), Government of India for providing fund to do this work and also thank to the anonymous reviewer.

\section{References}

Azekura K 1980 New finite element solution technique for neutron diffusion equations. J. Nucl. Sci. Technol. 17(2): 89-97

Bart M N, Jose A E, Nico S, Julio R B and Ashim K D 2011 Fuzzy finite element analysis of heat conduction problems with uncertain parameters. J. Food Eng. 103(1): 38-46

Biswas D, Ram K S and Rao S S 1976 Application of 'natural coordinate system' in the finite element solution of multigroup neutron diffusion equation. Ann. Nucl. Energy 3: 465-469

Chakraverty S and Nayak S 2012 Fuzzy finite element method for solving uncertain heat conduction problems. Coupled Syst. Mech. 1(4): 345-360 
Chakraverty S and Nayak S 2013a Non probabilistic solution of uncertain neutron diffusion equation for imprecisely defined homogeneous bare reactor. Ann. Nucl. Energy 62: 251-259

Chakraverty S and Nayak S 2013b Fuzzy finite element method in diffusion problems, Mathematics of uncertainty modelling in the analysis of engineering and science problems, IGI global, Chapter 15, 309328

Glasstone S and Sesonke A 2004 Nuclear reactor engineering. fourth edition, vol. 1, CBS Publishers and Distributors Private Limited

Nagaya Y and Mori T 2011 Calculation of effective delayed neutron fraction with Monte Carlo perturbation techniques. Ann. Nucl. Energy 38(2-3): 254-260

Nagaya Y, Chiba G, Mori T, Irwanto D and Nakajima K 2010 Comparison of Monte Carlo calculation methods for effective delayed neutron fraction. Ann. Nucl. Energy 37(2): 1308-1315

Nayak S and Chakraverty S 2013 Non-probabilistic approach to investigate uncertain conjugate heat transfer in an imprecisely defined plate. Int. J. Heat Mass Transfer 67: 445-454

Shi B and Petrovic B 2011 Implementation of the modified power iteration method to two-group Monte Carlo eigenvalue problems. Ann. Nucl. Energy 38(4): 781-787

Sjenitzer B L and Hoogenboom J E 2011 Variance reduction for fixed-source Monte Carlo calculations in multiplying systems by improving chain-length statistics. Ann. Nucl. Energy 38(2): 2195-2203

Yamamoto T 2012 Monte Carlo method with complex weights for neutron leakage-corrected calculations and anisotropic diffusion coefficient generations. Ann. Nucl. Energy 50: 141-149

Zadeh L A 1965 Fuzzy sets, information and control 8: 338-353 